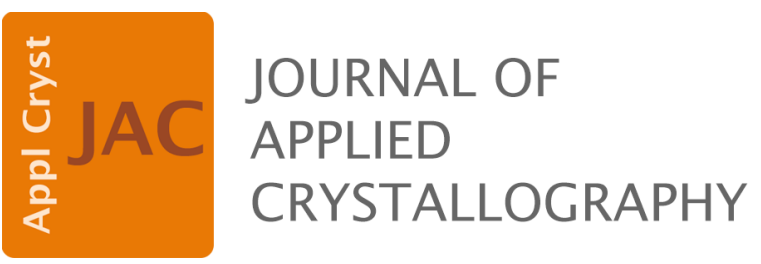

Volume 51 (2018)

Supporting information for article:

Correct interpretation of diffraction properties of quartz crystals for X-ray optics applications. Corrigendum

Xian-Rong Huang, Thomas Gog, Jungho Kim, Elina Kasman, Ayman H. Said, Diego M. Casa, Michael Wieczorek, Marcelo G. Hönnicke and Lahsen Assoufid 


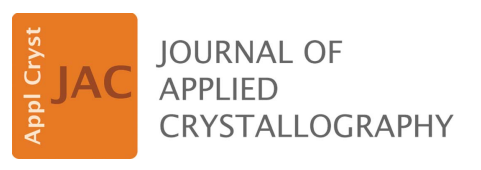

ISSN 1600-5767

Received 13 June 2017

Accepted 19 December 2017

Edited by S. Boutet, SLAC National Accelerator Laboratory, Menlo Park, USA

Keywords: X-ray optics; quartz; Bragg reflection; Darwin bandwidth; X-ray analyzers.

Supporting information: this article has supporting information at journals.iucr.org/j

\section{Correct interpretation of diffraction properties of quartz crystals for X-ray optics applications}

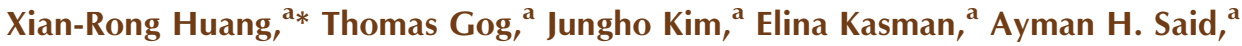 \\ Diego M. Casa, ${ }^{a}$ Michael Wieczorek, ${ }^{a}$ Marcelo G. Hönnicke ${ }^{b}$ and Lahsen Assoufid ${ }^{a}$
}

\begin{abstract}
aAdvanced Photon Source, Argonne National Laboratory, 9700 South Cass Avenue, Argonne, Illinois 60439, USA, and b Instituto de Ciencias da Vida e da Natureza, Universidade Federal da Integracao Latino-Americana, 2044 Foz do Iguacu, Parana, 85867-970, Brazil. *Correspondence e-mail: xiahuang@aps.anl.gov
\end{abstract}

Quartz has hundreds of strong Bragg reflections that may offer a great number of choices for making fixed-angle X-ray analyzers and polarizers at virtually any hard X-ray energies with selectable resolution. However, quartz crystals, unlike silicon and germanium, are chiral and may thus appear in two different forms of handedness that are mirror images. Furthermore, because of the threefold rotational symmetry along the c axis, the $\left\{h_{1} h_{2} h_{3} L\right\}$ and $\left\{h_{2} h_{1} h_{3} L\right\}$ Bragg reflections may have quite different Darwin bandwidth, reflectivity and angular acceptance, although they have the same Bragg angle. The design of X-ray optics from quartz crystals therefore requires unambiguous determination of the orientation, handedness and polarity of the crystals. The Laue method and single-axis diffraction technique can provide such information, but the variety of conventions used in the literature to describe quartz structures has caused widespread confusion. The current studies give detailed guidelines for design and fabrication of quartz X-ray optics, with special emphasis on the correct interpretation of Laue patterns in terms of the crystallography and diffraction properties of quartz. Meanwhile, the quartz crystals examined were confirmed by X-ray topography to have acceptably low densities of dislocations and other defects, which is the foundation for developing high-resolution quartz-based X-ray optics.

\section{Introduction}

Crystal-based X-ray optics, such as monochromators, analyzers, polarizers etc., are mainly fabricated from $\mathrm{Si}$ single crystals (or occasionally Ge which has higher X-ray absorption). Unfortunately, high-symmetry Si (or Ge) crystals have very limited Bragg reflections. For example, Si has only 48 different Bragg reflections for photon energy $E<16 \mathrm{keV}$ (see Table $\mathrm{S} 1$ in the supporting information). In Bragg diffraction ( $2 d \sin \theta=\lambda$ with a fixed $d$ spacing), one can usually change the Bragg angle $\theta$ to change the wavelength $\lambda$ (photon energy $E$ ) to desired values. However, there are special cases where one has little freedom to change $\theta$. A typical example is the nearback-reflection analyzer for inelastic X-ray scattering (IXS), where $\theta$ must be very close to $90^{\circ}$ (Burkel et al., 1987; Sinn et al., 2001; Ishikawa et al., 2015). Another example is an X-ray polarizer with $\theta=45^{\circ}$ that only diffracts $\sigma$-polarization X-rays (Alp et al., 2000). For such applications, the above 48 different $d$ values of Si can only provide 48 sparsely spaced discrete energies. This makes it difficult or impossible to make such Sibased X-ray optics work at arbitrary photon energies, particularly for resonant X-ray scattering experiments that require the photon energies to be close to various atomic absorption edges or emission lines (Gog et al., 2013; Ament et al., 2011). 
The other limitation of $\mathrm{Si}$ and $\mathrm{Ge}$ is that all the back reflections except 111 and 220 are multiple-beam diffraction with detrimental parasite reflections (Sutter et al., 2001; Huang et al., 2014).

A possible solution to avoid these problems is to use less symmetric crystals, such as quartz, sapphire, lithium niobate, silicon carbide etc. (Sutter et al., 2005). For example, Table S2 in the supporting information lists more than 700 different Bragg reflections of quartz $\left(\alpha-\mathrm{SiO}_{2}\right)$ with back-reflection Darwin reflectivity $R_{\max } \geq 0.3$. These reflections give about 420 different back-reflection energies that are almost continuously distributed in the medium energy range 5$16 \mathrm{keV}$. This indicates that one can use quartz to make nearback-reflection $\left(\theta \simeq 90^{\circ}\right)$ analyzers (or monochromators) for any photon energy $E>5 \mathrm{keV}$ (Ketenoglu et al., 2015; Honnicke et al., 2016; Sutter \& Yavaş, 2017). It also means that one can make quartz polarizers $\left(\theta \simeq 45^{\circ}\right)$ for arbitrary energies. Meanwhile, these reflections can be completely free of parasite reflections owing to the non-cubic crystal structure of quartz.

Unfortunately, most of these exotic crystals (particularly sapphire, lithium niobate and silicon carbide) have high densities of dislocations and other defects that are extremely detrimental to the resolution and efficiency of Bragg diffraction. However, Sutter et al. (2006) have demonstrated that synthetic quartz crystals grown from aqueous solutions have an acceptably low density of dislocations and other defects. The X-ray topographs taken from the samples studied in this paper, as well as measurements made by other laboratories, confirm this result. We have used such quartz crystals from

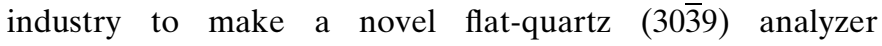
(combined with a multilayer collimating Montel mirror) that achieved a high resolution of $3.9 \mathrm{meV}$ (close to the theoretical value of $3.6 \mathrm{meV}$ ) in resonant inelastic X-ray scattering (RIXS) at $E=11.215 \mathrm{keV}$ (Kim et al., 2017). This bandpass is similar to the resolution of $4 \mathrm{meV}$ achieved on a large-area quartz wafer by Sutter et al. (2006) at $9.979 \mathrm{keV}$. For comparison, the best resolution of silicon analyzers obtained at the $\operatorname{Ir} L_{3}$ absorption edge is $25 \mathrm{meV}$ (Kim et al., 2014; Moretti Sala et al., 2013). These demonstrations, together with recent work from other groups (e.g. Honnicke et al., 2016), indicate that quartz-based X-ray optics may be very promising for high- to ultrahigh-resolution RIXS and IXS (from sub$10 \mathrm{meV}$ to sub-meV) in the future.

In contrast to the mature Si optics, developing quartz optics requires a complete set of new procedures for fabricating quartz crystals, from orienting to cutting/dicing, etching, polishing and characterization. In this paper we first give a relatively comprehensive description of the X-ray diffraction properties of quartz crystals that are associated with the complicated crystallographic structures and the two types of handedness. We concentrate on differentiation between the two types of lattice planes, $\left\{h_{1} h_{2} h_{3} L\right\}$ and $\left\{h_{2} h_{1} h_{3} L\right\}$, which have identical $d$ spacing but can have quite different Darwin bandwidths, angular acceptance and diffraction efficiency. Therefore, unambiguous differentiation between these confusable lattice planes is usually a critical starting point for correct fabrication of quartz optics with desired resolution and performance.

\section{Crystallographic properties of quartz}

$\alpha$-Quartz belongs to the trigonal crystal system with two types of handedness, the right-handed and left-handed structures with space groups $P 3_{2} 21$ (No. 154) and $P 3_{1} 21$ (No. 152), respectively (International Tables for Crystallography, 2016). Unfortunately, a variety of conventions have been used in the literature to describe the $\alpha$-quartz crystallography. For example, Donnay \& Le Page (1978) have summarized from the earlier literature eight conventions of quartz described in both the right- and left-handed hexagonal coordinates, but these conventions have caused more confusion and difficulties instead of leading to consistency. In particular, the use of the left-handed hexagonal coordinate system makes the lefthanded quartz structure incompatible with general X-ray crystallography principles because almost all crystal structure factors and X-ray diffraction are calculated in right-handed coordinate systems. Therefore, the right-handed hexagonal coordinate system is exclusively used in this paper to describe the unit cells of both left- and right-handed quartz structures. In the right-handed hexagonal coordinate system, the unit cell contains three Si atoms with fractional coordinates $(u, 0,0)$, $(-u,-u, 1 / 3)$ and $(0, u, 2 / 3)$ and six $\mathrm{O}$ atoms with fractional coordinates $(x, y, z),(y, x, 2 / 3-z),(-y, x-y, 2 / 3+z),(-x$, $y-x, 1 / 3-z),(y-x,-x, 1 / 3+z)$ and $(x-y,-y,-z)$ for right-handed quartz. Note that the three Si atoms form a lefthanded helix along the $\mathbf{c}[0001]$ axis, which is opposite to the 'optical handedness' (i.e. the direction the quartz crystal rotates the plane of polarized light) (Akhavan, 2012; Donnay \& Le Page, 1978). Here we adopt the data from Le Page \& Donnay (1976): $u=0.4699, x=0.4141, y=0.2681$ and $z=$ 0.1188 , together with the lattice constants $a=b=4.9134 \AA, c=$ $5.4052 \AA, \alpha=\beta=90^{\circ}$ and $\gamma=120^{\circ}$. The unit cell defined by these parameters corresponds to the $z(+)$ setting of righthanded quartz in the right-handed hexagonal coordinate system by Donnay \& Le Page (1978). We adopt this setting here because it seems to be the most frequently used setting in the literature, and it has also been adopted in the database of the software package XOP (Sanchez del Rio \& Dejus, 2004). Nevertheless, note that in addition to the eight conventions summarized by Donnay \& Le Page there are other conventions in the literature that describe the atom positions with different notations or different $u, x, y$ and $z$ values. The Laue patterns and diffraction properties of right-handed quartz presented in this paper and the supporting information were calculated strictly with the above setting and the specific $u, x, y$ and $z$ values. Accordingly, left-handed quartz has the space group $P 3_{1} 21$, which is a mirror structure of right-handed quartz (see §5). The Debye temperatures of the $\mathrm{O}$ and $\mathrm{Si}$ atoms in the quartz structure are 749.31 and $790.03 \mathrm{~K}$, which were calculated from the data provided by Le Page et al. (1980). In the following, the right-handed quartz structure is assumed if not explicitly specified. 
On the basis of the above right-handed structure, we developed a computer program that can automatically calculate the structure factors and the Darwin curves of all Bragg reflections of quartz and consequently can select the reflections satisfying any specific requirements (see the supporting information). Our program adopts the same atomic scattering factor database and the same algorithms for calculations of structure factors and Debye-Waller factors as the software package XOP (Sanchez del Rio \& Dejus, 2004). For example, in Table S2 of the supporting information we have used this program to produce the complete list of strong back reflections of quartz with Bragg energies $E_{\mathrm{BR}}<16 \mathrm{keV}$ and maximum Darwin reflectivity $R_{\max }>0.3$ at room temperature $(293.15 \mathrm{~K})$. One can conveniently use this table to design near-back-reflection quartz analyzers. In general, these Bragg reflections preserve high reflectivity also for non-back-reflection Bragg angles (with higher photon energies). Therefore, Table S2 also provides guidelines for choosing the suitable reflections for general diffraction of quartz. In addition, our program, which is freely available upon request, can be used to select all the Bragg reflections satisfying any criteria (e.g. Bragg angles, photon energies, reflectivity, Darwin bandwidths, angular acceptance or their combinations), such that the most suitable reflection(s) need never be neglected in quartz optics design.

The diffraction properties and principles revealed in Table S2 can be understood from the detailed quartz structure. Fig. 1 shows an idealized rendering of a quartz crystal with the three most common types of flat faces that can be naturally developed during growth: the $\mathbf{r}$ faces (major rhombohedrons) with Miller indices $\{01 \overline{1} 1\}$, the $\mathbf{z}$ faces (minor rhombohedrons) with Miller indices $\{10 \overline{1} 1\}$, and the prismatic $\mathbf{m}$ faces with Miller indices $\{10 \overline{1} 0\}$ and $\{01 \overline{1} 0\}$ (Akhavan, 2012; Donnay \& Le Page, 1978). Here the most prominent feature is that there is only threefold rotational symmetry along the c axis, as indicated by Fig. 1(b). It is the lack of sixfold symmetry along $\mathbf{c}[0001]$ that makes the $\mathbf{r}$ faces physically different from the $\mathbf{z}$ faces. For instance, the $\mathbf{r}$ faces of as-grown quartz crystals are usually larger than the $\mathbf{z}$ faces.
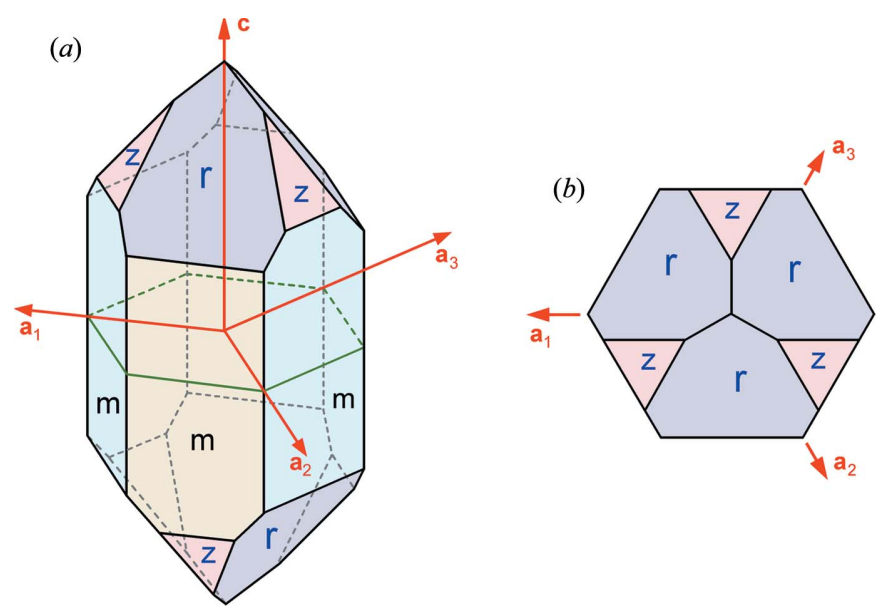

Figure 1

(a) Schematic of the idealized shape of the quartz crystal with the three types of common faces. (b) Top view of $(a)$.
Table 1

Equivalent lattice planes of $\left\{h_{1} h_{2} h_{3} L\right\}$ and $\left\{h_{2} h_{1} h_{3} L\right\}$ for $\alpha$-quartz.

$\left\{h_{1} h_{2} h_{3} L\right\}:\left(h_{1} h_{2} h_{3} L\right)\left(h_{2} h_{3} h_{1} L\right)\left(h_{3} h_{1} h_{2} L\right)\left(h_{2} h_{1} h_{3} \bar{L}\right)\left(h_{3} h_{2} h_{1} \bar{L}\right)\left(h_{1} h_{3} h_{2} \bar{L}\right)$ $\left\{h_{2} h_{1} h_{3} L\right\}:\left(h_{2} h_{1} h_{3} L\right)\left(h_{3} h_{2} h_{1} L\right)\left(h_{1} h_{3} h_{2} L\right)\left(h_{1} h_{2} h_{3} \bar{L}\right)\left(h_{2} h_{3} h_{1} \bar{L}\right)\left(h_{3} h_{1} h_{2} \bar{L}\right)$

More generally, the lack of the sixfold symmetry can make a Bragg reflection $\left\{h_{1} h_{2} h_{3} L\right\}$ [with $h_{3} \equiv-\left(h_{1}+h_{2}\right)$ ] different from the reflection $\left\{h_{2} h_{1} h_{3} L\right\}$ in X-ray diffraction, although the two reflections have the same Bragg angle owing to the fact that the two sets of lattice planes $\left(h_{1} h_{2} h_{3} L\right)$ and $\left(h_{2} h_{1} h_{3} L\right)$ have exactly the same $d$ spacing. Note that quartz also has twofold rotational symmetry along the a axes. On the basis of these symmetry operations, any $\left(h_{1} h_{2} h_{3} L\right)$ lattice plane has five equivalent planes, as listed in Table 1 . Here we use $\left\{h_{1} h_{2} h_{3} L\right\}$ to represent any of the six equivalent planes (or indices of the corresponding Bragg reflections). Similarly, $\left\{h_{2} h_{1} h_{3} L\right\}$ represents another six equivalent planes that are also listed in Table 1. In general, none of the $\left\{h_{1} h_{2} h_{3} L\right\}$ planes are equivalent to any of the $\left\{h_{2} h_{1} h_{3} L\right\}$ planes (for $h_{1} \neq h_{2}$ ), although all 12 planes have the same spacing $d$. Fig. 2 shows the intersections of the 12 lattice planes with the (0001) plane. These planes cut the $\mathbf{c}$ axis at $c / L$ or $-c / L$, which is not shown.

Figs. 3(a) and 3(b) show the calculated Darwin curves of Bragg reflections $\{30 \overline{3} 9\}$ and $\{03 \overline{3} 9\}$ with the same Bragg angle $\theta_{\mathrm{B}}=88.17^{\circ}$ for photon energy $E=11.215 \mathrm{keV}$. Here the two reflections have different bandwidths $\left(\Delta E_{\mathrm{BW}}=3.60\right.$ and $1.65 \mathrm{meV}$, respectively) and different peak reflectivity $\left(R_{\max }=\right.$ 0.68 and 0.42$)$. The angular Darwin width of $\{30 \overline{3} 9\}$ is $\Delta \theta_{\mathrm{FWHM}}=2.1^{\prime \prime}$, which is also different from that of $\{03 \overline{3} 9\}$, $0.96^{\prime \prime}$. Fig. 3(c) shows the experimentally measured rocking curves (solid lines) from symmetric $\{30 \overline{3} 9\}$ and $\{03 \overline{3} 9\}$ reflections. In these measurements, the incident beam was an undulator beam monochromated by an $\mathrm{Si}(111)$ double-crystal monochromator (DCM). Here the two rocking curves are much wider than the theoretical Darwin curves owing to the convolution of the instrumental functions (i.e. the finite bandwidth and divergence of the incidence beam). But the

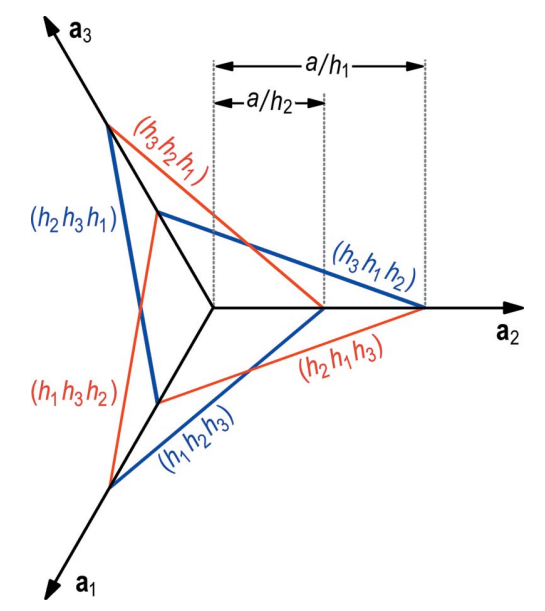

Figure 2

Intersection lines of lattice planes $\left\{h_{1} h_{2} h_{3} L\right\},\left\{h_{2} h_{1} h_{3} L\right\}$ and their equivalent planes with the (0001) plane in the hexagonal coordinate system. 
diffraction intensities clearly verify that the two reflections are not equivalent. More such $\left\{h_{1} h_{2} h_{3} L\right\}$ and $\left\{h_{2} h_{1} h_{3} L\right\}$ reflection pairs with different bandwidths and reflectivity can be found in Table S2.

For X-ray diffraction and optics applications of quartz, therefore, one must unambiguously differentiate the two types
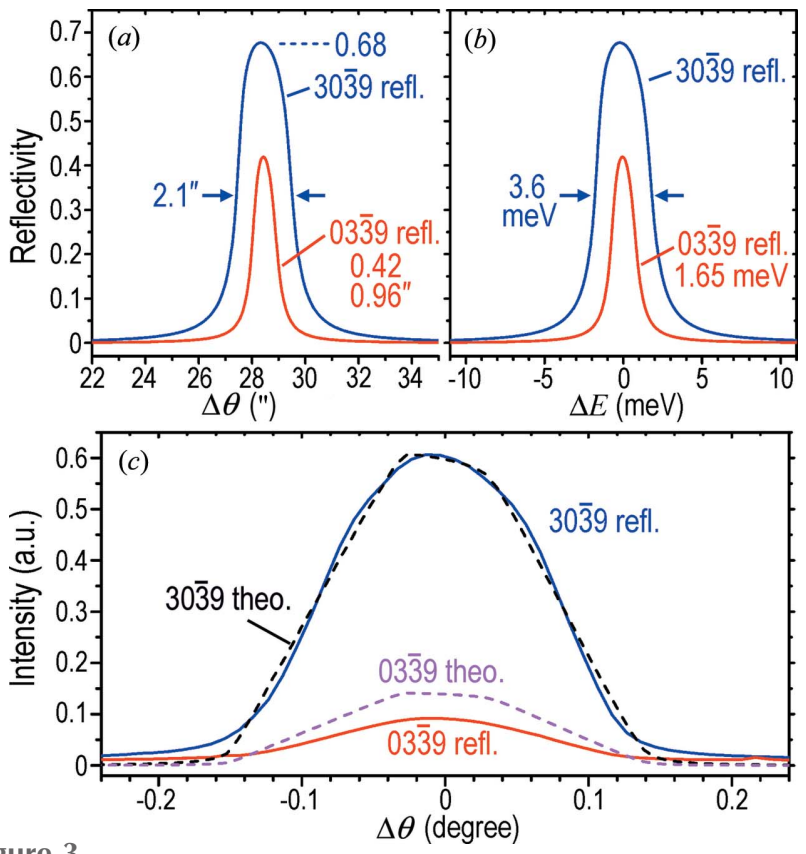

Figure 3

(a) Calculated angular Darwin curves of symmetric $30 \overline{3} 9$ and $03 \overline{3} 9$ reflections of right-handed quartz at $E=11.215 \mathrm{keV}$ and $\theta_{\mathrm{B}}=88.17^{\circ} . \Delta \theta=$ 0 corresponds to the Bragg angle $\theta_{\mathrm{B}}=88.17^{\circ}$, and the peak shift is due to the small X-ray refraction correction. (b) The corresponding calculated energy-dependent Darwin curves. $\Delta E=0$ corresponds to the Bragg energy $E=11.215 \mathrm{keV}$. (c) Experimentally measured rocking curves of the two reflections with an undulator beam monochromated by an $\mathrm{Si}(111)$ DCM. The dashed lines are the theoretical rocking curves that include the convolution of the DCM. $\sigma$-polarization.
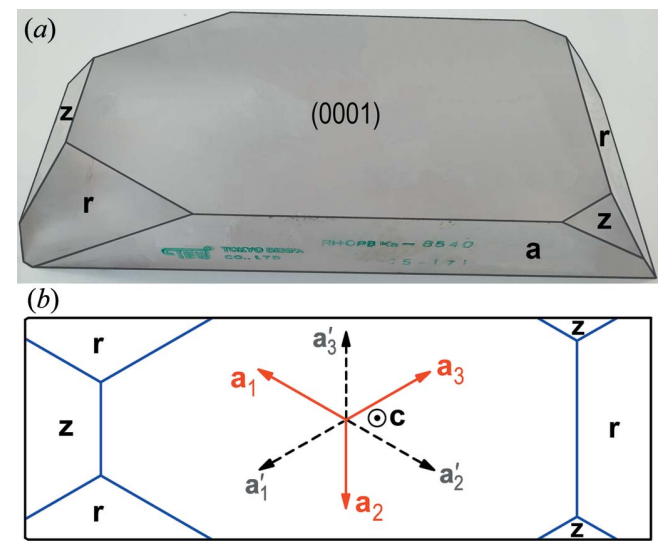

Figure 4

(a) Synthetic right-handed quartz crystal with natural flat $\mathbf{r}$ and $\mathbf{z}$ faces. The large $\mathbf{a}$ and $\mathbf{c}$ faces are cut surfaces. The sharp edges are highlighted by black lines. (b) Schematic of the top view of $(a)$. Note that the coordinate systems resulting from $\pm 120^{\circ}$ (threefold) rotation of the a axes about the c axis are equivalent. Crystal dimensions: $220 \times 85 \times 40 \mathrm{~mm}$ (thickness). of lattice planes. This is particularly important for making high-resolution quartz optics with resolution better than $10 \mathrm{meV}$, where the two types of reflections can give completely different bandwidths, efficiency and angular acceptance. In the following, we will illustrate how to determine the $\left\{h_{1} h_{2} h_{3} L\right\}$ and $\left\{h_{2} h_{1} h_{3} L\right\}$ planes in real crystals.

\section{Determination of quartz orientation by X-ray diffraction}

For developing quartz optics, we have acquired several large high-quality synthetic quartz crystals from Tokyo Denpa Co. Ltd, all of which have nearly the same shape as the crystal shown in Fig. 4(a). The crystal has naturally developed shiny $\mathbf{r}$ and $\mathbf{z}$ faces as well as a shiny (1010) face on the left side. The rough $\mathbf{a}$ and $\mathbf{c}$ faces are cut surfaces. Fig. 4(b) schematically shows the top view of the crystal.

We have cut and polished a few $(30 \overline{3} 9)$ wafers, with a thickness of $2 \mathrm{~mm}$, from these crystals for topography characterization. Fig. 5(a) is a white-beam transmission topograph taken from such a wafer. Here the large imaging volume, $3.7 \times$ $2.2 \times 2 \mathrm{~mm}$, only contains four short dislocation segments (black lines) with no other obvious defects, indicating that the crystal has very high crystalline quality. In fact, for the reflection geometry (the typical working geometry of X-ray monochromators, analyzers and polarizers) with a small X-ray penetration (extinction) depth of $\sim 10 \mu \mathrm{m}$, the reflection topography very rarely shows any dislocations or other defects on the surfaces of the wafers studied. For example, the $20 \overline{2} 6$ symmetric reflection topograph in Fig. 5(b) is completely dislocation free. These results agree with other groups' recent characterization work of quartz crystals acquired from the same company (Sutter et al., 2006; Hönnicke et al., 2013; personal communications with R. Verbeni at the European
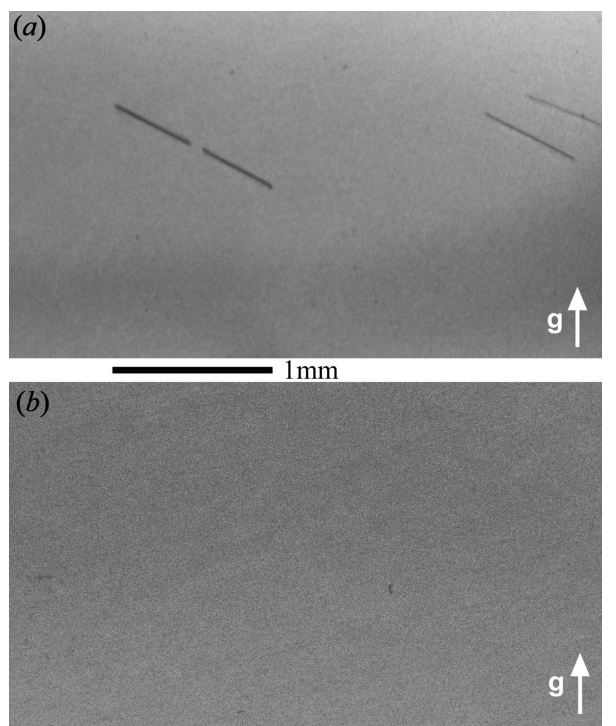

Figure 5

White-beam topography of a (30̄39) quartz crystal of thickness $2 \mathrm{~mm}$. (a) Transmission topograph. $\mathbf{g}=\overline{2} 022$ reflection with $E=14 \mathrm{keV}$. (b) Reflection topograph. Symmetric $\mathbf{g}=20 \overline{2} 6$ reflection with $E=$ $11.2 \mathrm{keV}$, penetration depth $\sim 14 \mu \mathrm{m}$. 
Synchrotron Radiation Facility). These crystals indicate the growing improvements of synthetic quartz when compared with earlier crystals reported in the literature (Lang \& Miuscov, 1967; Härtwig et al., 1977; Sutter et al., 2005). Therefore, our topography studies show that the latest quartz crystals are of sufficiently high quality for high-resolution Xray optics applications.

In order to identify the orientation of the crystal in Fig. 4, we have to correctly determine the right-handed hexagonal coordinate system on the crystal. For as-grown quartz crystals with naturally developed $\mathbf{r}$ and $\mathbf{z}$ faces, one can see from Fig. 1(b) that the crystallographic a axes are parallel to the projections of the intersection edges between the $\mathbf{r}$ and $\mathbf{z}$ faces onto the (0001) plane. On the basis of this principle, we can plot the a axes on the crystal in Fig. 4(b). However, we have two choices for the $\mathbf{a}$ axes: the $\mathbf{a}_{1}-\mathbf{a}_{2}-\mathbf{a}_{3}$ and $\mathbf{a}_{1}^{\prime}-\mathbf{a}_{2}^{\prime}-\mathbf{a}_{3}^{\prime}$ righthanded hexagonal coordinate systems that are rotated from each other by $60^{\circ}$. Here note that the assumed $\mathbf{r}$ and $\mathbf{z}$ faces are with respect to the $\mathbf{a}_{1}-\mathbf{a}_{2}-\mathbf{a}_{3}$ system. In the $\mathbf{a}_{1}^{\prime}-\mathbf{a}_{2}^{\prime}-\mathbf{a}_{3}^{\prime}$ system, the $\mathbf{r}$ faces ( $\mathbf{z}$ faces) would become $\mathbf{z}$ faces ( $\mathbf{r}$ faces). How can we distinguish between the $\mathbf{r}$ and $\mathbf{z}$ faces?

In our experiments, we have used the Laue method to study the crystal orientation in Fig. 4. Fig. 6(a) is a backward Laue pattern taken from the crystal in Fig. 4(a). In the experiment, an X-ray white beam from a $\mathrm{Cu}$ tube (operated at $30 \mathrm{kV}$ and $30 \mathrm{~mA}$ ) was incident normally on the (0001) surface and diffracted backward to a CCD camera a distance of $36 \mathrm{~mm}$ away. Fig. 6(b) is a computer simulated Laue pattern (Huang, 2010) overlapped on the recorded pattern. The simulation is based on the $\mathbf{a}_{1}-\mathbf{a}_{2}-\mathbf{a}_{3}$ system in Fig. 4(b) and the inset of Fig. 6(a). Obviously, the two sets of Laue patterns coincide with each other very well. This experimentally verifies that the $\mathbf{a}_{1}-\mathbf{a}_{2}-\mathbf{a}_{3}$ system in Fig. $4(b)$ is the correct choice and that the assumed $\mathbf{r}$ and $\mathbf{z}$ faces are correct.

The Laue pattern in Fig. 6(a) correctly shows that quartz only has threefold rotational symmetry along the $\mathbf{c}$ axis. For example, although the six spots indicated by the blue and green arrows in Fig. 6(b) are located at the sixfold symmetric positions (with $E=11.257 \mathrm{keV}$ and $\theta_{\mathrm{B}}=64.5^{\circ}$ ), the three $\{30 \overline{3} 8\}$ spots identified by the blue arrows have relatively strong intensities, while the three $\{03 \overline{3} 8\}$ spots identified by the green arrows are almost invisible in Fig. 6(a). This difference is consistent with our calculations. At $E=11.257 \mathrm{keV}$ and $\theta_{\mathrm{B}}=$ $64.5^{\circ}$, the $\{30 \overline{3} 8\}$ reflections have a theoretical bandwidth of $\Delta E_{\mathrm{BD}}=14.9 \mathrm{meV}$, an angular Darwin width of $\Delta \theta_{\mathrm{FWHM}}=$ $0.573^{\prime \prime}$ and a Darwin peak reflectivity of $R_{\max }=0.91$. By contrast, the three corresponding values of the $\{03 \overline{3} 8\}$ reflections are $\Delta E_{\mathrm{BD}}=1.7 \mathrm{meV}, \Delta \theta_{\mathrm{FWHM}}=0.065^{\prime \prime}$ and $R_{\max }=0.35$, which correspond to much weaker integrated intensity of the $\{03 \overline{3} 8\}$ spots in Fig. $6(a)$. This again shows that $\left\{h_{1} h_{2} h_{3} L\right\}$ is not equivalent to $\left\{h_{2} h_{1} h_{3} L\right\}$. Here, if one does not have the computer simulation capability, the three easily recognizable $\{30 \overline{3} 8\}$ diffraction spots in the (0001) backward Laue pattern of Fig. 6(a) can be conveniently used to determine the correct a axes, i.e. the three a axes are rotated by $30^{\circ}$ clockwise away from the three radial vectors from the center to the three $\{30 \overline{3} 8\}$ spots, respectively.
In our experiments, when we rotated the crystal by $180^{\circ}$ about the $\mathbf{a}_{2}$ axis to make Laue diffraction from the opposite $(000 \overline{1})$ face, we obtained the same Laue pattern as Fig. 6(a) (instead of a horizontally flipped pattern). From a similar simulation, we may obtain another right-handed hexagonal coordinate system $\mathbf{a}_{1}^{\prime \prime}-\mathbf{a}_{2}^{\prime \prime}-\mathbf{a}_{3}^{\prime \prime}-\mathbf{c}^{\prime \prime}$, with $\mathbf{a}_{1}^{\prime \prime}=\mathbf{a}_{3}, \mathbf{a}_{2}^{\prime \prime}=\mathbf{a}_{2}, \mathbf{a}_{3}^{\prime \prime}=\mathbf{a}_{1}$ and $\mathbf{c}^{\prime \prime}=-\mathbf{c}$ (not shown), or its equivalent $\pm 120^{\circ}$ rotations along $\mathbf{c}^{\prime \prime}$. However, this $\mathbf{a}_{1}^{\prime \prime}-\mathbf{a}_{2}^{\prime \prime}-\mathbf{a}_{3}^{\prime \prime}-\mathbf{c}^{\prime \prime}$ system is related to the $\mathbf{a}_{1}-\mathbf{a}_{2}-\mathbf{a}_{3}-\mathbf{c}$ system by the twofold rotational symmetry along the $\mathbf{a}_{2}$ axis, i.e. the two systems are equivalent. Thus, the Laue pattern taken from either the (0001) or (0001) face always yields the same or equivalent hexagonal coordinate system,
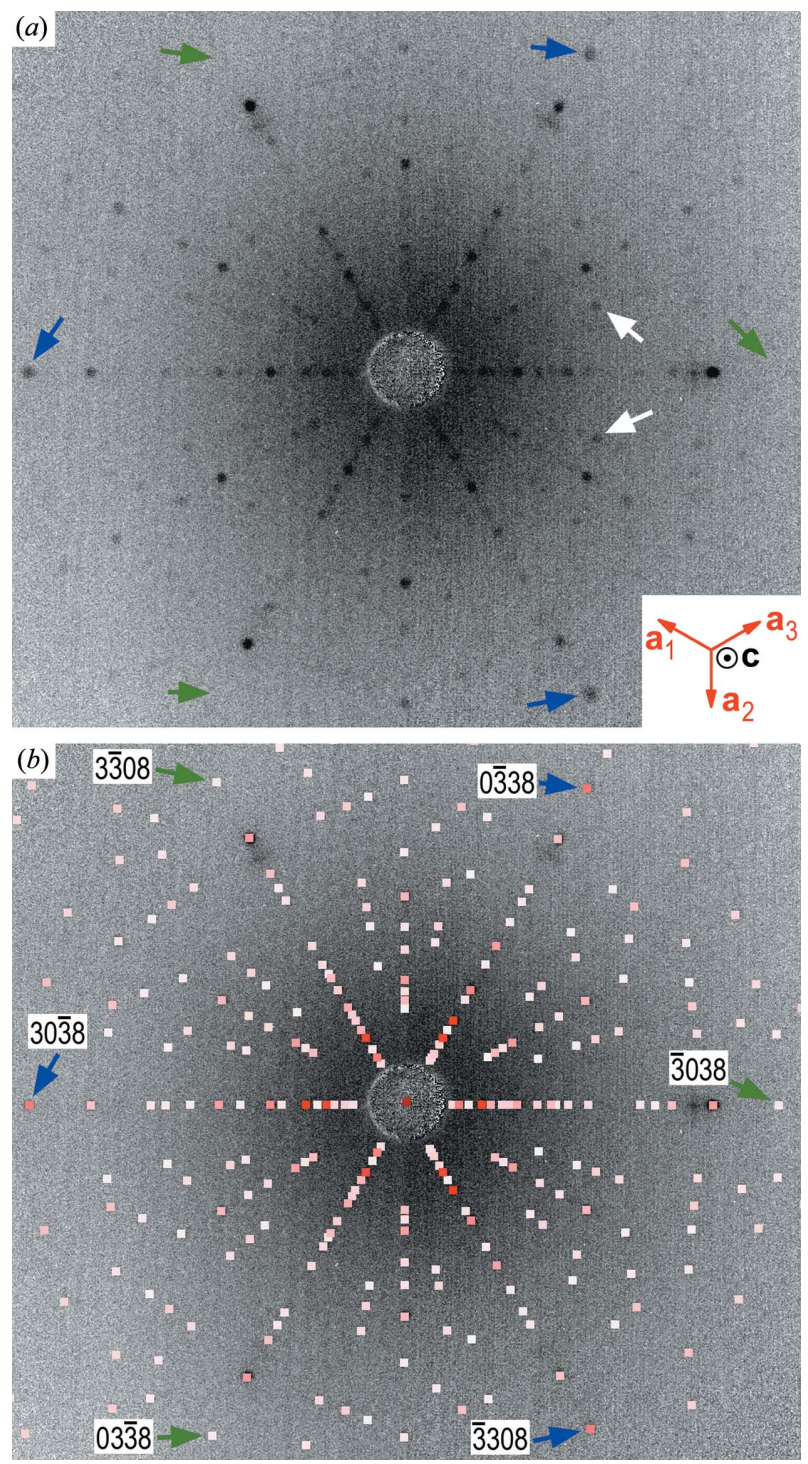

Figure 6

(a) Back-reflection Laue pattern taken from the (0001) face of the quartz crystal in Fig. 4(a). Note that the specific (12) 10$)$ a face in Fig. 4(a), which is also the mirror plane $A$ in Fig. S1 of the supporting information, is still perpendicular to the $\mathbf{a}_{2}$ axis here. (b) Simulated Laue pattern overlapped on the recorded one. The view direction is the same as that of Fig. 4(b), i.e. viewed from the backside of the CCD to the crystal surface along the $-\mathbf{c}$ direction. The range of colors of the spots from red to white indicates the decrease of intensity. Image width $94 \mathrm{~mm}$. 
and $\mathbf{a}_{1}^{\prime}, \mathbf{a}_{2}^{\prime}$ and $\mathbf{a}_{3}^{\prime}$ in Fig. 4(a) can never be mistaken as the positive crystallographic a axes on the basis of the Laue patterns. (Meanwhile, this principle also means that quartz is a nonpolar structure along the [0001] direction.) Therefore, $\mathrm{X}$-ray Laue diffraction can independently identify the accurate orientations of quartz without relying on the crystal shapes or faces.

After determination of the orientation by the Laue pattern, we cut a $(30 \overline{3} 9)$ wafer and a $(03 \overline{3} 9)$ wafer from the quartz crystal shown in Fig. 4(a). These two wafers were ground, etched and polished with identical procedures. Subsequently, we performed rocking curve measurements of the two wafers in the symmetric reflection geometry with an incident synchrotron beam from an Si(111) DCM, as mentioned in $\$ 2$. The two rocking curves measured by rotating the quartz wafers are shown in Fig. 3(c), where the $30 \overline{3} 9$ reflection is, indeed, much stronger than the $03 \overline{3} 9$ reflection. This again confirms the crystallographic a axes we have determined in Fig. 4(b). Note that the intrinsic Darwin widths of the two reflections are much narrower in Fig. 3(a). In Fig. 3(c), significant broadening of the two measured rocking curves is mainly owing to the finite bandwidth and divergence of the incident beam from the DCM. More specifically, the rocking curves are broadened by the weak dependence of the wavelength on the diffraction angle when the Bragg angle is close to $90^{\circ}$.

The dashed lines in Fig. 3(c) are the simulated rocking curves that include the convolution of the DCM and the divergence of the white beam (15 $\mu \mathrm{rad})$ incident on the DCM. Here the $30 \overline{3} 9$ diffraction intensities have been normalized to the measured intensities. The measured rocking curve width of $0.177^{\circ}$ is nearly the same as the simulation. With the same normalization factor, however, the measured $03 \overline{3} 9$ diffraction intensities are noticeably lower than the theoretical intensities. One possible reason for the discrepancies may be the extreme sensitivity of quartz to thermal load, which is a severe disadvantage of quartz optics. Our experiments show that the direct beam from the $\mathrm{Si}(111) \mathrm{DCM}$ (with a bandwidth of $\sim 1.5 \mathrm{eV}$ ) can remarkably broaden the rocking curve of quartz and reduce the reflectivity. In the experiment of Fig. 3(c), the incident beam was reduced by a chopper to $2.1 \%$ of the original intensity, but the reduced heat load may still be too high for quartz to work normally. Therefore, applications of quartz for synchrotron X-ray monochromators will require careful heat load management, although this is not necessary for quartz analyzers.

Here note that the purpose of this simple experiment is solely for distinguishing between the $(30 \overline{3} 9)$ and $(03 \overline{3} 9)$ wafers from the integrated diffraction intensities, and it is not intended for high-resolution measurements of the meV-level bandwidths of the two reflections. In separate experiments, we have used a four-bounce symmetric $\mathrm{Si}(844)$ monochromator (with a bandwidth of $\sim 8 \mathrm{meV}$ ) after the $\mathrm{Si}(111) \mathrm{DCM}$ to further monochromatize the incident beam. Nearly theoretical rocking curves of the $30 \overline{3} 9$ reflection from the quartz wafer were repeatedly measured using this $8 \mathrm{meV}$ monochromatic beam without thermal problems. In addition, we have achieved a deconvoluted energy resolution of the $30 \overline{3}$ 9 reflection of $3.9 \mathrm{meV}$ from the entire three-inch (3039) wafer in a novel RIXS setup, which is very close to the theoretical value of $3.6 \mathrm{meV}$ (Kim et al., 2017). These results clearly demonstrate the feasibility of developing (ultra)high-resolution quartz analyzers in the medium-energy range.

\section{Difference between $h_{1} h_{2} h_{3} L$ and $\bar{h}_{1} \bar{h}_{2} \bar{h}_{3} \bar{L}$ reflections}

Quartz has no polarity along the $\mathbf{c}$ axis owing to the twofold rotational symmetry along the a axes. However, since the structure does not have centrosymmetry, it can be polar along other directions. Meanwhile, the atomic scattering factor of $\mathrm{Si}$, $f_{\mathrm{Si}}$, has a significant imaginary part in the low- to mediumenergy range, which can cause anomalous scattering. For example, at $E=6,8,10,12,14$ and $16 \mathrm{keV}$, the values of $\operatorname{Im}\left(f_{\mathrm{Si}}\right)$ are $0.57,0.33,0.22,0.15,0.11$ and 0.085 , respectively. Consequently, the non-negligible imaginary part of $f_{\mathrm{Si}}$ can cause the breakdown of Friedel's law for the noncentrosymmetric quartz crystal, i.e. $\left|F\left(\mathbf{g}=h_{1} h_{2} h_{3} L\right)\right| \neq\left|F\left(-\mathbf{g}=\bar{h}_{1} \bar{h}_{2} \bar{h}_{3} \bar{L}\right)\right|$, where $F$ denotes the structure factor. As presented in Table S2, most of the $h_{1} h_{2} h_{3} L$ and $\bar{h}_{1} \bar{h}_{2} \bar{h}_{3} \bar{L}$ reflection pairs (Friedel pairs) indeed have nonzero Bragg peak reflectivity differences $\Delta R_{\max }$ caused by the anomalous scattering effect. The differences can be quite remarkable for a number of reflections, such as $11 \overline{2} 0,11 \overline{2} \overline{1}, 3 \overline{1} 22,3 \overline{124}, 3 \overline{1} 25,4 \overline{22} 7,7 \overline{344}, 7 \overline{34} 5$ and $8 \overline{177}$. These reflections have $\left|\Delta R_{\max }\right|>0.1$ with respect to their opposite reflections $-\mathbf{g}$, and the relative reflectivity difference is even larger. However, except for the reflectivity difference, a reflection pair $h_{1} h_{2} h_{3} L$ and $\bar{h}_{1} \bar{h}_{2} \bar{h}_{3} \bar{L}$ always have the same bandwidth $\Delta E_{\mathrm{BW}}$ and angular Darwin width $\Delta \theta_{\mathrm{FWHM}}$.

As an example, Fig. 7 shows the calculated Darwin curves of reflections $11 \overline{2} 1$ and $\overline{11} 2 \overline{1}$ at $E=8.05 \mathrm{keV}$ ( $\mathrm{Cu} \mathrm{K \alpha _{1 }}$ radiation). Here the two curves indeed have the same Darwin width, $\Delta \theta_{\text {FWHM }}=1.26^{\prime \prime}$, but the two peak reflectivity values differ by $\Delta R_{\max }=-0.145$, or $\left|\Delta R_{\max } / R_{\max }(\overline{11} 2 \overline{1})\right|=20 \%$. Such a difference is measurable in high-resolution $\mathrm{X}$-ray diffraction

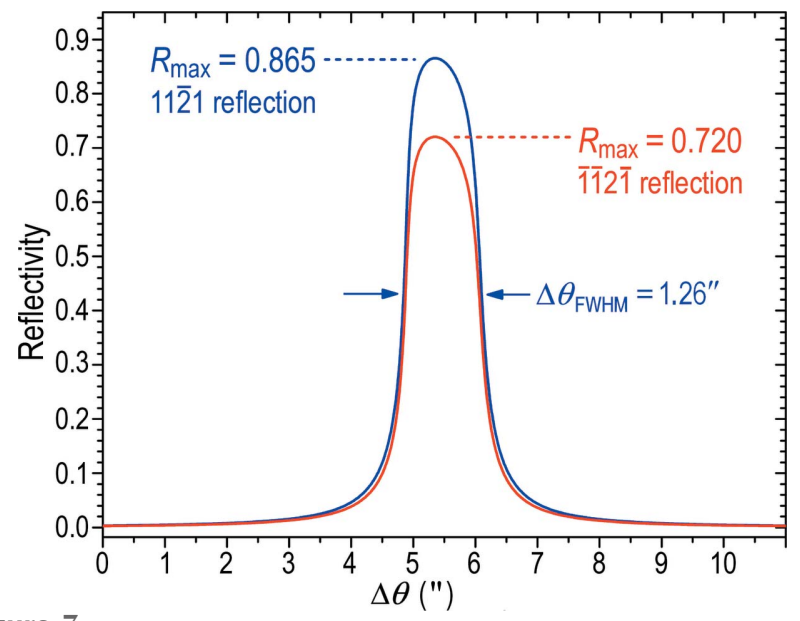

Figure 7

Calculated Darwin curves of quartz $11 \overline{2} 1$ and $\overline{11} 2 \overline{1}$ reflections. $E=$ $8.05 \mathrm{keV}, \theta_{\mathrm{B}}=20.15^{\circ}, \sigma$-polarization. 
(or double-crystal diffraction) from the two faces of a doubleside-polished (112 1 ) plate of quartz (De Vries, 1958).

Therefore, for those Friedel reflection pairs with significant $\Delta R_{\max }$, one must distinguish between the positive and negative surfaces, $h_{1} h_{2} h_{3} L$ and $\bar{h}_{1} \bar{h}_{2} \bar{h}_{3} \bar{L}$, of a quartz crystal (particularly for a parallel-sided plate) during crystal fabrication. In general, the surface corresponding to larger reflectivity should be used for X-ray diffraction to achieve better efficiency. Nevertheless, $\Delta R_{\max }$ is small for most reflections of quartz, which implies that differentiation between the $h_{1} h_{2} h_{3} L$ and $\bar{h}_{1} \bar{h}_{2} \bar{h}_{3} \bar{L}$ surfaces may be unnecessary for these cases. Here one can use Table S2 as guidelines even for non-backreflection geometry.

The upper spot indicated by a white arrow in Fig. 6(a) is the $\mathbf{g}=21 \overline{3} 11$ reflection. With respect to the horizontal central line, the $21 \overline{3} 11$ spot has a mirror spot in the lower part that is the $3 \overline{1} \overline{2} 11$ reflection (identified by the other white arrow). Here note that the $3 \overline{1} \overline{2} 11$ reflection is equivalent to $\overline{2} \overline{1} 3 \overline{11}=-\mathbf{g}$. This phenomenon is true for all the other reflections, i.e. each reflection (g) spot in the upper part of the (0001) Laue pattern in Fig. 6 has its negative reflection $(-\mathbf{g})$ spot symmetrically located in the lower part. Since $\Delta R_{\max }$ is small or negligible for most reflections of quartz, the (0001) backward Laue pattern should be nearly mirror symmetric with respect to the horizontal central line, which is obviously true in Fig. 6(a). But note that the Laue pattern has no mirror symmetry about the vertical central line.

\section{Left-handed quartz}

The left-handed quartz structure is a mirror image of righthanded quartz. Therefore, the unit cell of left-handed quartz can be simply obtained by mirroring the right-handed atom positions $\left(x_{i}, y_{i}, z_{i}\right)$ with respect to any planes. For example, the simplest transformation is $\left(x_{i}, y_{i}, z_{i}\right) \rightarrow\left(x_{i}, y_{i},-z_{i}\right)$ with respect to the (0001) plane, or $\left(x_{i}, y_{i}, z_{i}\right) \rightarrow\left(y_{i}, x_{i}, z_{i}\right)$ with respect to the $\{1 \overline{1} 00\}$ plane. As shown in Fig. S1 of the supporting information, we choose to mirror the right-handed structure to the left-handed one with respect to the (1210) plane, $A$ in Fig. S1 [parallel to the a face in Fig. 4(a)], by the transformation $\left(x_{i}, y_{i}, z_{i}\right) \rightarrow\left(x_{i}, x_{i}-y_{i}, z_{i}\right)$, followed by a partial translation of $-\mathbf{c} / \mathbf{3}$. Here the translation does not change the crystal structure. Thus, the overall transformation is $\left(x_{i}, y_{i}, z_{i}\right) \rightarrow\left(x_{i}, x_{i}-y_{i}, z_{i}-1 / 3\right)$. Applying this operation on the right-handed quartz structure in $\$ 2$, we find that the three $\mathrm{Si}$ atoms of the left-handed unit cell are located at $(u, u$, $-1 / 3),(-u, 0,0)$ and $(0,-u, 1 / 3)$, while the six $O$ atoms are located at $(x, x-y, z-1 / 3),(y, y-x, 1 / 3-z),(-y,-x, 1 / 3+$ $z),(-x,-y,-z),(y-x, y, z)$ and $(x-y, x, 2 / 3-z)$, in the same right-handed hexagonal coordinate system (still with $u=$ $0.4699, x=0.4141, y=0.2681$ and $z=0.1188$ ). This unit cell corresponds to the $z(-)$ setting of left-handed quartz in the right-handed hexagonal coordinate system of Donnay \& Le Page (1978). The benefit of choosing this special left-handed unit cell (LH) is that any one of its structure factors is related to that of the right-handed unit cell $(\mathrm{RH})$ by

$$
F\left(h_{1} h_{2} h_{3} L_{\mathrm{LH}}\right) \equiv F\left(\bar{h}_{1} \bar{h}_{2} \bar{h}_{3} \bar{L}_{\mathrm{RH}}\right) .
$$

This relationship indicates that X-ray diffraction from any $\left(h_{1} h_{2} h_{3} L\right)$ lattice plane of left-handed quartz is always equivalent to diffraction from the opposite side of the corresponding lattice plane, $\left(\bar{h}_{1} \bar{h}_{2} \bar{h}_{3} \bar{L}\right)$, of right-handed quartz.

Note that the two kinds of unit-cell configurations are defined in the same right-handed $\mathbf{a}_{1}-\mathbf{a}_{2}-\mathbf{a}_{3}-\mathbf{c}$ coordinate system. Therefore, if we perform Laue diffraction on the lefthanded (0001) quartz surface, we will obtain a Laue pattern similar to Fig. 6 with the same a and $\mathbf{c}$ axes, except that each reflection $h_{1} h_{2} h_{3} L_{\mathrm{RH}}$ in Fig. 6 will be replaced by reflection $h_{1} h_{2} h_{3} L_{\mathrm{LH}}$. According to equation (1), $h_{1} h_{2} h_{3} L_{\mathrm{LH}}$ is equivalent to $\bar{h}_{1} \bar{h}_{2} \bar{h}_{3} \bar{L}_{\mathrm{RH}}$. On the other hand, we have proved in $\S 4$ that each $h_{1} h_{2} h_{3} L_{\mathrm{RH}}$ spot in the upper part of Fig. 6 has a mirror sport in the lower part that is equivalent to $\bar{h}_{1} \bar{h}_{2} \bar{h}_{3} \bar{L}_{\mathrm{RH}}$. Thus, the replacement of each reflection $h_{1} h_{2} h_{3} L_{\mathrm{RH}}$ in Fig. 6 by $\bar{h}_{1} \bar{h}_{2} \bar{h}_{3} \bar{L}_{\mathrm{RH}}\left(\equiv h_{1} h_{2} h_{3} L_{\mathrm{LH}}\right)$ simply results in a vertically flipped pattern of Fig. 6. Therefore, the Laue pattern of the left-handed (0001) quartz surface is simply a vertically flipped pattern of the right-handed (0001) surface.

However, we have demonstrated above that, for most reflections of quartz, the reflectivity difference between the $h_{1} h_{2} h_{3} L$ and $\bar{h}_{1} \bar{h}_{2} \bar{h}_{3} \bar{L}$ reflections is small or even negligible, i.e. the Laue pattern in Fig. 6 is almost symmetric with respect to the central horizontal line. Thus, the vertically flipped pattern, which is the Laue pattern of left-handed quartz, is nearly the same as the original pattern of the right-handed quartz in Fig. 6. In other words, if the reflectivity difference $\Delta R_{\max }$ between a reflection pair $h_{1} h_{2} h_{3} L$ and $\bar{h}_{1} \bar{h}_{2} \bar{h}_{3} \bar{L}$ is negligible, which is true for most reflections of quartz, it is usually unnecessary to distinguish between the two types of handedness.

In some special cases where $\Delta R_{\max }$ cannot be ignored, one must identify the handedness of the quartz crystal, which can usually be determined by the natural crystal shapes (Akhavan, 2012), by the optical rotation method (Herschel, 1822) or by the etch patterns (Van Dyke, 1940). From the above descriptions, one can also use X-ray anomalous scattering to identify the handedness (De Vries, 1958). This method consists of three steps. First, the a axes can be determined from the backward Laue pattern of the (0001) surface, independent of the handedness (see Figs. 4 and 6). Second, on the basis of the determined $\mathbf{a}$ and $\mathbf{c}$ axes, one can cut a (11̄21) plate, followed by identical double-side polishing. Third, one can perform high-resolution rocking curve measurements from the two surfaces $(11 \overline{2} 1)$ and ( $\overline{11} 2 \overline{1}$ ) (for example, at $E=8.05 \mathrm{keV}$ or lower). From Fig. 7, if the measured Bragg intensity of the $11 \overline{2} 1$ reflection from the front surface (1121) is higher than that from the back surface (112 $\overline{1})$, the crystal is of right handedness. Otherwise, the crystal is of left handedness.

\section{Summary}

We have demonstrated that the $\alpha$-quartz crystal has hundreds to thousands of Bragg reflections in the medium-energy range, compared to only a few tens of reflections for silicon. The numerous reflections of quartz have various applications in 
X-ray optics, particularly for making high- to ultrahigh-resolution near-back-reflection analyzers and fixed-diffractionangle X-ray polarizers at any energy. To design and fabricate quartz-based X-ray optics, however, one must understand the diffraction properties of the large number of Bragg reflections and their relationships to the crystallographic structures. For this purpose, we have used both theoretical calculations and experiments to study the crystal symmetry of quartz, the equivalent Bragg reflections, the bandwidth and reflectivity of each reflection, etc. In particular, we have shown the use of the Laue method (including simulation) to unambiguously identify the crystallographic a and $\mathbf{c}$ axes of quartz crystals. Only after such accurate determination of the crystal orientation can one correctly cut the $\left(h_{1} h_{2} h_{3} L\right)$ surface without confusion with the $\left(h_{2} h_{1} h_{3} L\right)$ surface. Then we theoretically and experimentally demonstrated the difference between the $h_{1} h_{2} h_{3} L$ and $h_{2} h_{1} h_{3} L$ reflection pairs (including their different bandwidths, reflectivity and angular acceptance) and illustrated the importance of differentiation between these two types of reflections in fabrication of quartz X-ray optics.

We have also illustrated the anomalous scattering effect of quartz, which can give rise to remarkable reflectivity differences between some of the $h_{1} h_{2} h_{3} L$ and $\bar{h}_{1} \bar{h}_{2} \bar{h}_{3} \bar{L}$ Friedel reflection pairs. For applications of these reflections, one must correctly distinguish between the front surface $\left(h_{1} h_{2} h_{3} L\right)$ and the opposite surface $\left(\bar{h}_{1} \bar{h}_{2} \bar{h}_{3} \bar{L}\right)$ of a quartz crystal. Meanwhile, it has been proved that any Bragg reflection of left-handed quartz is exactly equivalent to the opposite reflection of righthanded quartz, i.e. $h_{1} h_{2} h_{3} L_{\mathrm{LH}} \equiv \bar{h}_{1} \bar{h}_{2} \bar{h}_{3} \bar{L}_{\mathrm{RH}}$. For the Bragg reflections with significant anomalous scattering, therefore, one must also identify the handedness of the quartz crystal, which can be achieved by measuring the reflectivity difference between the $11 \overline{2} 1$ and $\overline{11} 2 \overline{1}$ reflection pairs. For those reflections with small or negligible anomalous scattering (which should be verified by dynamical-theory calculations), it is usually unnecessary to differentiate between the polarity of the Bragg planes and the handedness of the quartz.

Finally, most of the general diffraction principles of quartz illustrated above may also apply to sapphire (space group $R \overline{3} c$ ) and lithium niobate (space group $R 3 c$ ), which likewise have trigonal crystal symmetry.

\section{Acknowledgements}

XRH and EK are grateful to A. T. Macrander at beamline 1BM of APS and J. Härtwig at the European Synchrotron Radiation Facility for great help in the white-beam topography work. TG would like to thank N. Sinclair for his help with the rocking curve measurements at beamline 35-ID of
APS. We also thank N. Pereira of Ecopulse for stimulating discussions.

\section{Funding information}

This work was supported by the US Department of Energy, Office of Science, Office of Basic Energy Sciences, under contract No. DE-AC-02-06CH11357.

\section{References}

Akhavan, A. C. (2012). The Quartz Page, http://www.quartzpage.de/. Alp, E. E., Sturhahn, W. \& Toellner, T. S. (2000). Hyperfine Interact. 125, 45-68.

Ament, L. J. P., van Veenendaal, M., Devereaux, T., Hill, J. \& van den Brink, J. (2011). Rev. Mod. Phys. 83, 705-767.

Burkel, E., Peisl, J. \& Dorner, B. (1987). Europhys. Lett. 3, 957-961.

De Vries, A. (1958). Nature, 181, 1193.

Donnay, J. D. H. \& Le Page, Y. (1978). Acta Cryst. A34, 584-594.

Gog, T., Casa, D. M., Said, A. H., Upton, M. H., Kim, J., Kuzmenko, I., Huang, X. \& Khachatryan, R. (2013). J. Synchrotron Rad. 20, 7479.

Härtwig, J., Dietrich, B., Bötger, R. \& Lübke, G. (1977). Phys. Status Solidi (A), 43, K131-K133.

Herschel, J. F. W. (1822). Trans. Camb. Philos. Soc. 1, 43-52.

Honnicke, M. G., Bianco, L. M., Ceppi, S. A., Cusatis, C., Huang, X., Cai, Y. Q. \& Stutz, G. E. (2016). J. Appl. Cryst. 49, 1443-1453.

Hönnicke, M. G., Huang, X., Cusatis, C., Koditwuakku, C. N. \& Cai, Y. Q. (2013). J. Appl. Cryst. 46, 939-944.

Huang, X. R. (2010). J. Appl. Cryst. 43, 926-928.

Huang, X.-R., Jia, Q., Wieczorek, M. \& Assoufid, L. (2014). J. Appl. Cryst. 47, 1716-1721.

International Tables for Crystallography (2016). Vol. A, Space-Group Symmetry, edited by M. I. Aroyo, 2nd online ed. Chester: International Union of Crystallgraphy.

Ishikawa, D., Ellis, D. S., Uchiyama, H. \& Baron, A. Q. R. (2015). J. Synchrotron Rad. 22, 3-9.

Ketenoglu, D., Harder, M., Klementiev, K., Upton, M., Taherkhani, M., Spiwek, M., Dill, F.-U., Wille, H.-C. \& Yavaş, H. (2015). J. Synchrotron Rad. 22, 961-967.

Kim, J., Casa, D., Said, A., Kim, B. J., Kasman, E., Huang, X. \& Gog, T. (2017). Unpublished.

Kim, J., Daghofer, M., Said, A. H., Gog, T., van den Brink, J., Khaliullin, G. \& Kim, B. J. (2014). Nat. Commun. 5, 4453.

Lang, A. R. \& Miuscov, V. F. (1967). J. Appl. Phys. 38, 2477-2483.

Le Page, Y., Calvert, L. D. \& Gabe, E. J. (1980). J. Phys. Chem. Solids, 41, 721-725.

Le Page, Y. \& Donnay, G. (1976). Acta Cryst. B32, 2456-2459.

Moretti Sala, M., Henriquet, C., Simonelli, L., Verbeni, R. \& Monaco, G. (2013). J. Electron Spectrosc. Relat. Phenom. 188, 150-154.

Sanchez del Rio, M. \& Dejus, R. J. (2004). Proc. SPIE, 5536, 171-174.

Sinn, H., Alp, E. E., Alatas, A., Barraza, J., Bortel, G., Burkel, E., Shu, D., Sturhahn, W., Sutter, J. P., Toellner, T. S. \& Zhao, J. (2001). Nucl. Instrum. Methods Phys. Res. A, 467-468, 1545-1548.

Sutter, J., Alp, E., Hu, M., Lee, P., Sinn, H., Sturhahn, W., Toellner, T., Bortel, G. \& Colella, R. (2001). Phys. Rev. B, 63, 094111.

Sutter, J. P., Baron, A. Q. R., Ishikawa, T. \& Yamazaki, H. (2005). J. Phys. Chem. Solids, 66, 2306-2309.

Sutter, J. P., Baron, A. Q. R., Miwa, D., Nishino, Y., Tamasaku, K. \& Ishikawa, T. (2006). J. Synchrotron Rad. 13, 278-280.

Sutter, J. P. \& Yavaş, H. (2017). arXiv:1612.07049 [physics.ins-det].

Van Dyke, K. S. (1940). Proc. IRE, 28, 399-406. 